\title{
Identifying the Forest Surfaces Prone to Fire Ignition and Wildfire Spread in Metropolitan Areas; a Comparative Case from Western Balkans ${ }^{\dagger}$
}

\author{
Artan Hysa \\ Faculty of Architecture and Engineering, Epoka University, Rr. Tiranë-Rinas, Km. 12, 1032 Vorë, Tirana, \\ Albania; ahysa@epoka.edu.al \\ † Presented at TERRAenVISION 2019, Barcelona, Spain, 2-7 September, 2019. \\ Published: 25 October 2019
}

\begin{abstract}
Human activity combined with the dynamics of severe climate conditions are accepted the main drivers of wildfire events in the Mediterranean region. This fact is urging for further comprehensive research focusing on the wildland-urban interface (WUI) at metropolitan scale, at which the tension between the cause and effect of wildfire is the highest. In this context, the study brings a comparative case between two metropolitan areas from Western Balkan countries, the forest lands of which are classified by their index of wildfire ignition probability (WIPI) and wildfire spreading capacity (WSCI). Originally, both indexing methods rely on a multi-criteria evaluation which considers simultaneously the geophysical, hydrometeorological and anthropogenic factors of the territory. All stages of the process are performed by utilizing QGIS software. First, the forest surfaces within the metropolitan zone of Tirana (AL) and Sarajevo (BH) are extracted from Urban Atlas land cover data being provided as an open source by Copernicus data portal (EU). Reference points grid (distance of $100 \mathrm{~m}$ ) overlapping with the forest surfaces serve as pivot points to which the relative values of each criteria are projected. Later the absolute values are normalized into 10 classes via Jenks natural break method. The class value of each criterion is introduced into the indexing equation multiplied by the unique impact factor being weighted via pairwise comparative method in Analytical Hierarchy processing. The majority of the workflow steps are automated via Graphical Modeler in QGIS utilizing open source spatial data, giving floor to further applicability of the method to similar cases. As a result, there are produced statistical and graphical information being useful for identifying wildfire prone forest surfaces within the metropolitan areas. Being applied into two different study areas, the results enable a comparative discussion and evaluation at regional scale. By utilizing open source software and data, this work contributes in the development of practical and re-applicable models of wildfire risk assessment promoting open access scientific culture. Finally, the study results successful in testing a rapid and cost free method for identifying the forest areas prone to wildfire ignition and spreading risk in metropolitan areas in support to disaster risk reduction agendas and sustainable Development Goals.
\end{abstract}

Keywords: disaster risk reduction; wildfire ignition probability index (WIPI); wildfire spreading capacity index (WSCI); forest fire; wildland urban interface (WUI)

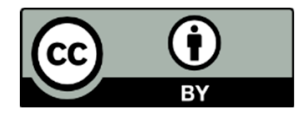

(C) 2019 by the authors. Licensee MDPI, Basel, Switzerland. This article is an open access article distributed under the terms and conditions of the Creative Commons Attribution (CC BY) license (http://creativecommons.org/licenses/by/4.0/). 